\title{
Charles Bonnet Syndrome after Occipital Infarction
}

\author{
Jung Seok Lee , KeunHyuk Ko ${ }^{*}$ Jung-Hwan Oh ${ }^{\star}$, Ho Kyu Lee ${ }^{\dagger}$, Joong-Goo Kim ${ }^{\star}$ \\ Departments of Neurology ${ }^{\star}$, Radiology ${ }^{\dagger}$, Jeju National University College of Medicine, Jeju, Korea
}

Visual hallucinations may occur in association with damage to any part of the visual pathway. Charles Bonnet syndrome (CBS) is characterized by vivid visual hallucinations caused by injury to the visual system, while other cognitive functions are preserved. We report on the clinical and neuro-imaging findings of two patients with CBS after acute stroke. The lesions were located bilaterally in the occipital lobes of both patients, although the lesion in patient 1 extended beyond the occipital lobe. In patient 1 , moving hallucinations persisted for 9 months after symptom onset, while in patient 2 , static hallucinations completely disappeared 7 days after the first hallucination. The latter case had a more extensive lesion on brain magnetic resonance imaging. Our cases suggest that the clinical course of CBS is variable irrespective of cortical lesion size, although this must be confirmed with more patients in the future.

J Neurosonol Neuroimag 201\&;10(2):154-157

Key Words: Visual hallucinations; Charles-Bonnet syndrome; Occipital lobe

Visual hallucinations may occur in association with damage to any part of the visual pathway. Charles Bonnet syndrome (CBS) is characterized by the presence of complex visual hallucinations in psychologically normal people. ${ }^{1}$ CBS has been reported in association with ocular disease ${ }^{2,3}$ and brain disease. ${ }^{4}$ There have been numerous articles concerning CBS associated with ocular disease. However, there have been relatively limited cases reported on CBS associated with ischemic stroke., ${ }^{5,6}$ We present the clinical course and brain magnetic resonance imaging (MRI) findings of two patients with CBS following bilateral ischemic injury in the occipital lobe.

\section{CASE REPORT}

\section{Patient 1}

A 68-year-old Korean woman with a history of hypertension and type 2 diabetes mellitus suddenly began to see strange apparitions in her left visual field. These vi-
Received: October 22, 2018

Revised: November 4, 2018

Accepted: November 5, 2018

Address for correspondence: Joong-Goo Kim

Department of Neurology, Jeju National University Hospital, 15 Aran 13-gil, Jeju 63241, Korea

Tel: +82-64-717-1620

Fax: $+82-64-717-1630$

E-mail: 1ilis1118@naver.com sions consisted of a white tumbler and a woman wearing white clothes. At first, these visual hallucinations appeared only on rare occasions, but the duration and frequency of the hallucinations increased over time. At times, she also saw visions of a short boy holding a duster. These visions always appeared in the left visual field. Whenever she looked to the left, the hallucination moved to the far left side and decreased in size. Because the symptoms persisted, the patient sought the assistance of an exorcist after 1 month. After the first exorcism, the visions of the tumbler-shaped object and woman wearing white clothes disappeared. However, she began to have visions of a black snake and these visions occurred less frequently than the former objects. After 3 weeks, the patient again received help from the exorcist because of the apparent effectiveness of the first exorcism. However, after the second exorcism, the black snake became enlarged and changed into a blue monster with a mustache. Three months after the onset of these symptoms, the patient was admitted and assessed. On neurologic examination, her consciousness was clear, and her visual acuity and color vision were 
all normal. Goldmann perimetry showed complete left hemianopsia with homonymous right inferior partial quadrantic defects adjacent to the vertical meridian (Fig. 1A). Brain fluid-attenuated inversion recovery (FLAIR) images demonstrated an old infarction in the right occipital lobe and an ischemic lesion in the left cuneus (Fig. 1B). Cognitive function was completely normal, and the patient did not take any medication other than for hypertension and diabetes. There were no abnormalities in the laboratory evaluation performed at the early stage of hospitalization. There was no evidence of central nervous system infection. In addition, no significant epileptiform discharge was shown in the initial electroencephalography (EEG). The patient was started on olanzapine at a dose of $10 \mathrm{mg} /$ day. A few days later, the frequency of the visual hallucinations was remarkably reduced, and she was discharged ten days after admission. However, the visual hallucinations continued to appear on rare occasions 6 months after her initial admission.

\section{Patient 2}

A 68-year-old Korean man developed complex visual hallucinations in the right visual field. These hallucinations included images of strange plants that had green leaves and white flowers. These plants persistently appeared in his right visual field and decreased in size whenever he looked to the right side. The patient was admitted and assessed 3 days after the onset of these symptoms. The patient had no history of previous neurological or psychological disorders. Baseline laboratory tests were normal. On neurologic examination, his consciousness was clear, and his visual acuity and color vision were normal. Humphrey perimetry revealed right congruent homonymous hemianopsia (Fig. 2A). There were no other sensory or motor deficits. Diffusion and FLAIR images obtained 3 days after symptom onset
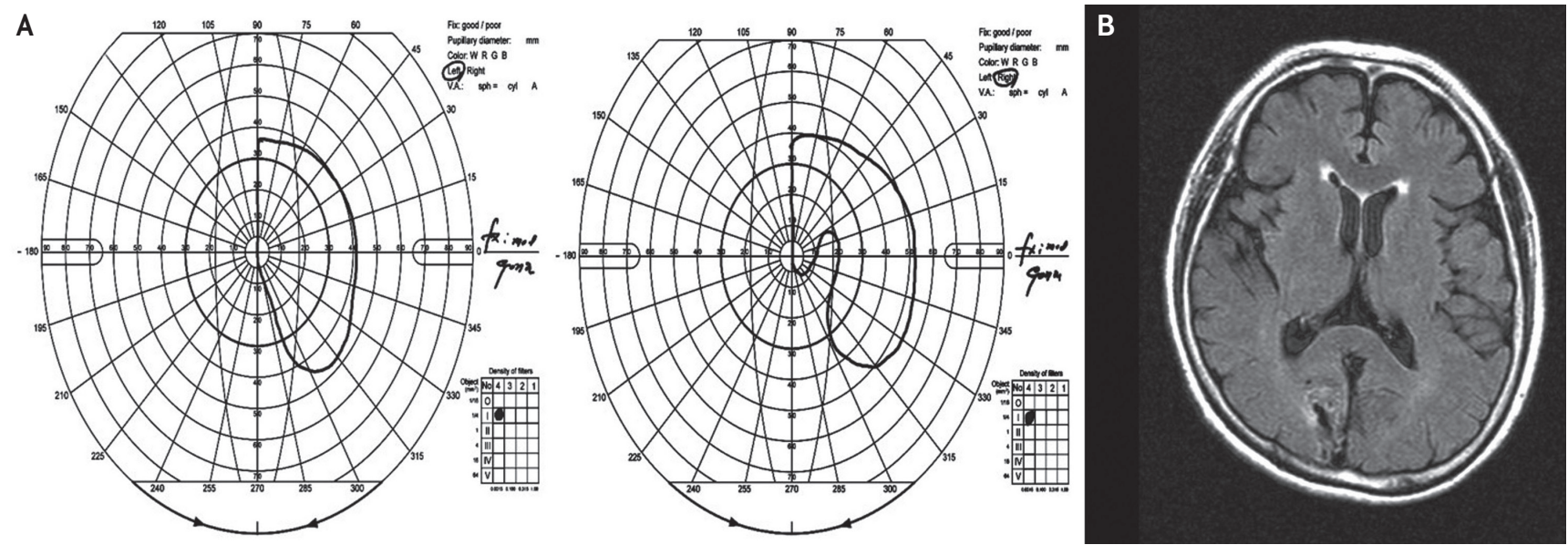

FIG. 1. (A) Goldmann perimetry of patient 1 showing complete left hemianopsia with homonymous right inferior partial quadrantic defect adjacent to the vertical meridian. (B) fluid-attenuated inversion recovery image of patient 1 showed old infarction in the right occipital lobe.
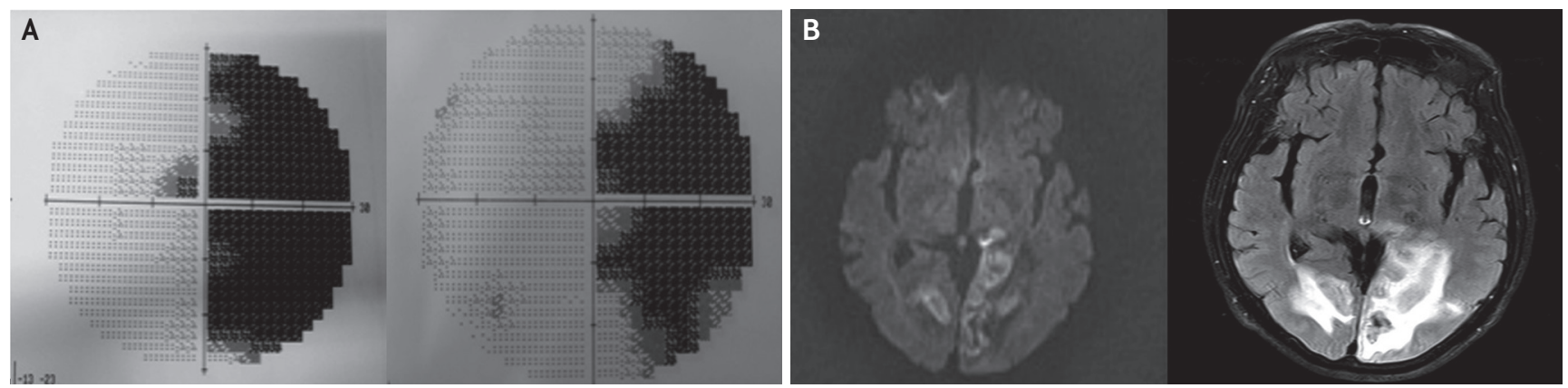

Fig. 2. (A) Humphrey perimetry of patient 2 showing right congruent homonymous hemianopsia. (B) Initial diffusion and fluid-attenuated inversion recovery image of patient 2 showed the hemorrhagic transformation of acute ischemic lesions in bilateral occipital lobes. 
showed hemorrhagic transformation of acute bilateral ischemic lesions in the occipital lobes (Fig. 2B). Initial EEG did not show a significant epileptiform discharge. Although without specific medical treatment, all of the hallucinations completely disappeared 7 days after the first hallucination, with the right congruent homonymous hemianopsia remaining unchanged.

\section{DISCUSSION}

CBS is characterized by vivid visual hallucinations caused by injury to the visual system while other cognitive functions are preserved. It is closely related to ocular diseases such as macular degeneration, diabetic retinopathy, glaucoma, and corneal diseases. ${ }^{1}$ CBS can also result from direct injury to the cerebral cortex, such as occipital lobe infarction, ${ }^{5,6}$ and occipital cortical and other cerebral resections. ${ }^{4}$ In our patients, CBS was believed to have resulted from direct injury to the occipital cortex for the following reasons: 1 ) they had no past medical history of psychological or neurological disorders and 2) the visual hallucinations occurred suddenly in the absence of hallucinations involving other sensory organs.

In CBS due to ocular lesions, visual hallucinations have not been shown to be confined to the area of the visual field defect. ${ }^{2,3}$ A previous study that examined visual hallucinations in patients with macular degeneration demonstrated that $30.8 \%$ of patients experienced visual hallucinations occurring outside the visual field defect. $^{2}$ Another study described a patient with central retinal artery occlusion who experienced visual hallucinations originating just outside the right border of the affected visual field. ${ }^{3}$

In CBS due to central lesions, visual hallucinations may occur only within an abnormal visual field. ${ }^{7}$ One report described a patient with left occipital lobe infarction and an old right posterior circulation infarct affecting the occipital region but sparing the primary visual cortex who experienced right homonymous hemianopsia, accompanied by complex visual hallucination in this region. ${ }^{6}$ Furthermore, a case series reported on 16 patients (not referred to as patients with CBS) who experienced complex visual hallucination in the hemianopic or quadrantanopic field. ${ }^{8}$ All of these patients had an occipital lesion on cranial computed tomography, although some had a parietal and temporal extension. Another report described four patients with postoperative brain lesions that experienced complex visual hallucinations in visual field defects. ${ }^{8}$ Both of our patients developed complex visual hallucinations following bilateral occipital damage. Visual hallucinations only appeared on the contralateral side of the more severely injured occipital cortex and were localized to the visual field defect area.

In our patients, the hallucinated images decreased in size when the patient looked to the side of the hallucinations. This condition has not been previously described. These facts suggest that eye movement might have some effect on the size of the hallucinated image. It is possible that voluntary saccade ${ }^{9}$ and pursuit eye movement ${ }^{10}$ show activation in occipital cortex, including the occipito-parietal or occipitotemporal junction.

The pathogenesis of CBS has not yet been ascertained. Visual hallucinations are thought to be the result of a release phenomenon in the ventral temporo-occipital cortex or the phenomenon of "denervation hypersensitivity". 7 Complex visual hallucinations in our patients may have been provoked by interference of the dorsal occipitoparietal processing system, which is related to the guidance of actions and recognition of objects in space. Moreover, the ventral temporo-occipital processing stream is involved with object recognition and form representation. ${ }^{7}$ Neurologists may potentially analyze complex visual hallucinations as part of the associated visual symptom of occipital lobe epilepsy (OLE). However, complex and vivid visual hallucinations were rarely reported in OLE patients. ${ }^{1}$

CBS has a variable disease course. It can either spontaneously disappear after days or continue for months. Patient 1 experienced extremely colorful and vivid moving hallucinations that reappeared over a 9-month period whereas patient 2 experienced static hallucinations that completely disappeared seven days after the first hallucination. Patient 1 received olanzapine, which was effective. In patient 2, the visual hallucinations spontaneously subsided, and the patient had more extensive lesions than patient 1 . It has been postulated that the extent of damage in the occipital, parietal, or temporal lesions is less decisive than the extent of neural activation resulting from the lesion. ${ }^{8}$ However, there have 
been no reports on the correlation between lesion size and the duration of visual hallucinations. Our cases show that lesion size and duration of visual hallucinations are inconsistent. In conclusion, our cases suggest that the clinical course of Charles Bonnet syndrome is variable irrespective of cortical lesion size, although this must be confirmed with more patients in the future.

\section{Acknowledgements}

This work is attributed to and was solely supported by the Department of Neurology, Jeju National University Hospital, Jeju, Korea.

\section{Conflicts of Interest}

No potential conflict of interest relevant to this article was reported.

\section{REFERENCES}

1. Teeple RC, Caplan JP, Stern TA. Visual hallucinations: differential diagnosis and treatment. Prim Care Companion J Clin Psychiatry. 2009;11:26-32.

2. Holroyd S, Rabins PV, Finkelstein D, Nicholson MC, Chase GA, Wisniewski SC. Visual hallucinations in patients with macular degeneration. Am J Psychiatry. 1992;149:1701-1706.

3. Tan CS, Sabel BA, Goh KY. Visual hallucinations during visual recovery after central retinal artery occlusion. Arch Neurol. 2006;63:598-600.

4. Freiman TM, Surges R, Vougioukas VI, Hubbe U, Talazko J, Zentner J, et al. Complex visual hallucinations (Charles Bonnet syndrome) in visual field defects following cerebral surgery. Report of four cases. J Neurosurg. 2004;101:846-853.

5. Beniczky S, Kéri S, Vörös E, Ungureán A, Benedek G, Janka $Z$, et al. Complex hallucinations following occipital lobe damage. Eur J Neurol. 2002;9:175-176.

6. Ashwin PT, Tsaloumas MD. Complex visual hallucinations (Charles Bonnet syndrome) in the hemianopic visual field following occipital infarction. J Neurol Sci. 2007;263:184-186.

7. Burke W. The neural basis of Charles Bonnet hallucinations: a hypothesis. J Neurol Neurosurg Psychiatry. 2002;73:535541.

8. Kölmel HW. Complex visual hallucinations in the hemianopic field. J Neurol Neurosurg Psychiatry. 1985;48:29-38.

9. Law I, Svarer C, Rostrup E, Paulson OB. Parieto-occipital cortex activation during self-generated eye movements in the dark. Brain. 1998;121(Pt 11):2189-2200.

10. Tanabe J, Tregellas J, Miller D, Ross RG, Freedman R. Brain activation during smooth-pursuit eye movements. Neuroimage. 2002;17:1315-1324. 